\title{
Complicaciones de la nefrectomía laparoscópica
}

\author{
Castillo O, Bejarano C, Cortés O, Pinto I, Hoyos J, Vitagliano G. \\ Unidad de Urología, Clinica Santa María. Departamento de Urología, Facultad de Medicina, \\ Universidad de Chile. Santiago, Chile.
}

Actas Urol Esp. 2006;30(8):812-818

\section{RESUMEN}

\section{COMPLICACIONES DE LA NEFRECTOMÍA LAPAROSCÓPICA}

Objetivos: Presentamos las complicaciones en nuestra serie de nefrectomía laparoscópica y el manejo dado a cada una de ellas.

Métodos: Entre noviembre de 1992 y marzo de 2004, 319 pacientes fueron llevados a cirugía renal ablativa con técnica laparoscópica: 152 mujeres y 167 hombres, con edad promedio de 45 años (rango: 0,5 - 82 años). Se realizaron 70 nefrectomías radicales, 16 nefroureterectomías radicales, 63 nefrectomías parciales, 124 nefrectomías simples, 15 nefroureterectomías simples, 8 heminefrectomías y 23 nefrectomías de donante vivo para trasplante.

Resultados: La tasa de complicaciones fue del 5,64\%. Las complicaciones más frecuentes fueron sangrado $(2,5 \%)$ y hematoma retroperitoneal $(1,2 \%)$. No hubo diferencia estadísticamente significativa en la tasa de complicaciones por sexo, edad o vía de abordaje: transperitoneal o lumboscópica. El realizar una técnica laparoscópica pura o con asistencia manual, no representó cambio en la tasa de complicaciones. No hubo casos de mortalidad en la serie.

Conclusiones: Nuestra serie de nefrectomía laparoscópica muestra una baja tasa de complicaciones. Consideramos que en manos experimentadas, la laparoscopía es una técnica segura en pacientes que requieren cirugia renal ablativa.

Palabras clave: Laparoscopia. Nefrectomía. Complicaciones.

\section{ABSTRACT \\ COMPLICATIONS IN LAPAROSCOPIC NEPHRECTOMY}

Objective: To report the complications and their respective management in our series of laparoscopic nephrectomy.

Material and methods: Between November 1992 and March 2004, 319 patients underwent laparoscopic ablative renal surgery: 152 were women and 167 were men, mean age was 45 years (range: 0,5 82 years). There were 70 radical nephrectomies, 16 radical nephroureterectomies, 63 partial nephrectomies, 124 simples nephrectomies, 15 simples nephroureterectomies, 8 heminephrectomies and 23 living donor nephrectomies.

Results: The complication rate was 5.64\%. The most frequent complications were bleeding (2.5\%) and retroperitoneal haematoma (1.2\%). There was not statically difference in complications rate by age, sex, or approach via: transperitoneal versus retroperitoneal. Complication rate was alike with laparoscopic or hand assisted technique. There was not mortality in the series.

Conclusions: We have a low complication rate in laparoscopic nephrectomy. We believe that laparoscopy is a safe technique for patients that require ablative renal surgery when the surgical team has experience in advanced laparoscopic surgery. 
$\mathrm{D}$ esde el primer caso de nefrectomía laparoscópica comunicado en la literatura por Clayman et al. en $1991^{1}$ hasta la actualidad, se ha logrado ganar experiencia en cirugia renal laparoscópica, perfeccionando las técnicas quirúrgicas, haciendo que estos procedimientos sean de baja morbilidad, con ventaja significativa frente a la cirugía abierta en dolor postoperatorio, estancia hospitalaria y período de incapacidad, así como un mayor grado de satisfacción para los pacientes.

La cirugia renal laparoscópica ha evolucionado a tal punto, que en centros con experiencia en esta técnica, se ha convertido en el procedimiento de elección para abordar entidades renales benignas y malignas. Sin embargo, al igual que en cirugía abierta convencional, se debe estar familiarizado con las complicaciones derivadas del procedimiento y de la técnica quirúrgica, buscando prevenirlas, reconocerlas oportunamente y manejarlas de forma adecuada. En nuestro grupo, la laparoscopia es la técnica de elección en cirugía renal. Presentamos las complicaciones en nuestra serie de cirugía renal laparoscópica: nefrectomía simple, heminefrectomía, nefroureterectomía simple, nefrectomía de donante vivo, nefrectomía radical, nefrectomía parcial y nefroureterectomía radical, y el manejo dado a cada una de ellas.

\section{MATERIAL Y MÉTODOS}

Se incluyen todos los pacientes operados con técnica laparoscópica por patología renal benigna o maligna que requirieron cirugía renal de tipo ablativo. Los procedimientos incluidos por patología benigna son: nefrectomía simple, heminefrectomía y nefroureterectomía simple; por patología tumoral: nefrectomía radical, nefrectomía parcial y nefroureterectomía radical. También se incluyen las nefrectomías de donante vivo para trasplante. Los datos fueron obtenidos de un registro prospectivo de todos los pacientes con patología renal operados por laparoscopia en nuestra Unidad desde noviembre de 1992 a marzo del 2004.

Las complicaciones se analizaron de acuerdo al sexo, edad, procedimiento quirúrgico, técnica: laparoscopia pura o con asistencia manual, vía de abordaje: transperitoneal o lumboscópica, tipo de complicación: en el acceso, derivadas del neumoperitoneo o propias del procedimiento. Los datos fueron analizados utilizando el software Stata versión 5.0 y como prueba estadística se usó chi $^{2}$ y el test exacto de Fisher cuando las celdas tenían un valor menor a 5 .

En todos los casos se usó profilaxis antibiótica en el momento de la inducción anestésica y soportes de compresión graduada en miembros inferiores como medida antiembólica. La técnica quirúrgica de la nefrectomía ha sido previamente descrita ${ }^{2-4}$.

Para los procedimientos laparoscópicos por vía transperitoneal el paciente se posiciona en decúbito lateral, previamente al abordaje se coloca sonda naso-gástrica y sonda vesical. Se realiza el neumoperitoneo cerrado, haciendo la punción con la aguja de Veress a nivel subcostal o en la fosa iliaca. De no lograrse una punción exitosa o ante la presencia de factores que impliquen alto riesgo de adherencias se realiza neumoperitoneo con técnica abierta. En la disección y movilización del riñón, se utiliza tijera con coagulación monopolar o el bisturí ultrasónico. El espécimen quirúrgico se coloca en una bolsa de polietileno y se extrae por una incisión en fosa ilíaca en casos de patología tumoral, o se expone el cuello de la bolsa por uno de los puertos y se realiza morcelación mecánica con una pinza tipo Foester (Fig. 1).

En el abordaje retroperitoneal (lumboscópico), el paciente se ubica en posición de lumbotomía clásica. A través de una incisión de 1 a $2 \mathrm{~cm}$ en

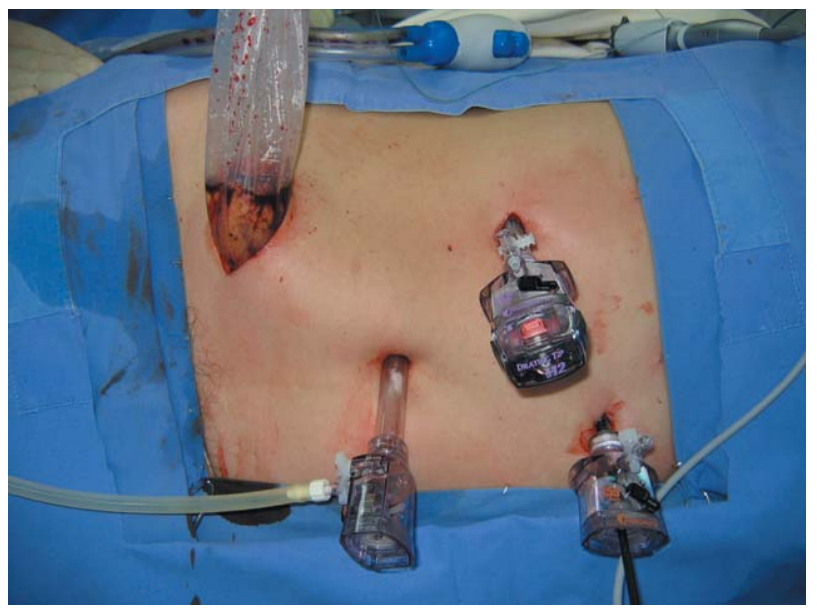

FIGURA 1. Posición de paciente y ubicación de trócares en nefrectomía parcial laparoscópica izquierda. Extracción de la pieza quirúrgica en bolsa de polietileno través de una incisión en la fosa iliaca. 
el vértice de la duodécima costilla, se divulsionan los planos musculares hasta la aponeurosis lumbo dorsal, la cual se incide, dando paso al retroperitoneo. El espacio de trabajo se obtiene con un balón hecho con el dedo medio de un guante quirúrgico № 8 anudado sobre una sonda Nelaton $18 \mathrm{Fr}$, el cual se distiende con solución salina hasta $800 \mathrm{~mL}$. Una vez creado este espacio, se introduce un trócar de $10 \mathrm{~mm}$ y bajo visión directa se ubican dos o tres trócares para los instrumentos de trabajo.

En la técnica con asistencia manual, el cirujano introduce su mano no dominante por una incisión en la fosa iliaca, y se crean dos puertos adicionales: uno para la óptica y otro para los instrumentos de trabajo. (Fig. 2).

En todos los procedimientos participó el autor principal, en su mayoría encabezando el equipo quirúrgico y en algunos casos, asistiendo y guian-
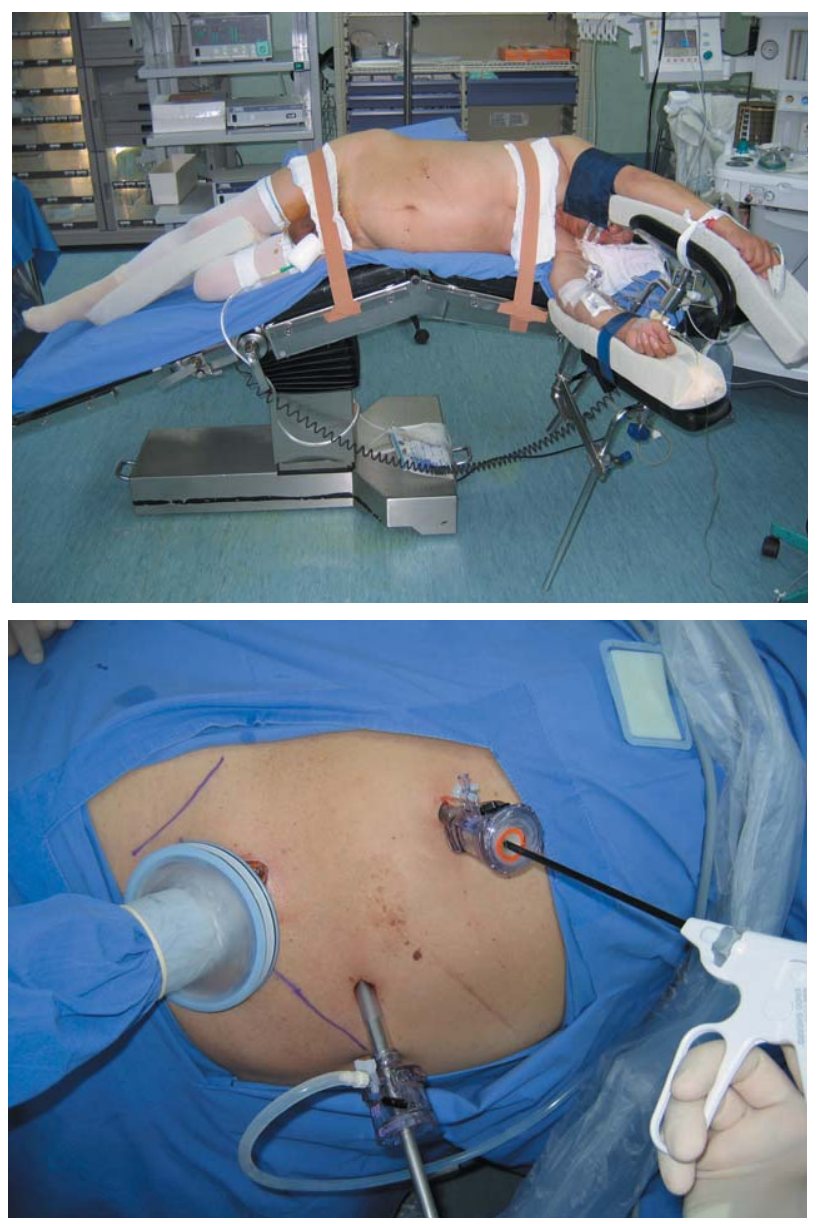

FIGURA 2. Posición de paciente y ubicación de trócares en nefrectomía laparoscópica derecha con asistencia manual. do a otros cirujanos en proceso de entrenamiento. Los demás integrantes del grupo: ayudantes, anestesiólogos, arsenalera y enfermeras, cuentan con amplia experiencia en cirugía laparoscópica y fueron, al igual que el cirujano, partícipes y colaboradores en todos los casos informados en esta serie.

\section{RESULTADOS}

Entre noviembre de 1992 y marzo de 2004, 319 pacientes fueron llevados a cirugía renal ablativa con técnica laparoscópica: 152 mujeres y 167 hombres, con edad promedio de 45 años (rango: 0,5 - 82 años). En 149 pacientes la indicación quirúrgica fue por patología tumoral, en este grupo se practicaron 70 nefrectomías radicales, 16 nefroureterectomías radicales y 63 nefrectomías parciales. Por entidades no oncológicas se indicaron 147 procedimientos: 124 nefrectomías simples, 15 nefroureterectomías y 8 heminefrectomías. Se realizaron 23 nefrectomías de donante vivo para trasplante.

\section{Nefrectomía radical}

Se practicaron 70 nefrectomías radicales, todas realizadas por vía transperitoneal: 38 pacientes operados con técnica laparoscópica pura y 32 con asistencia manual. En este grupo se presentaron 5 complicaciones: dos de ellas intraoperatorias y tres en el postoperatorio inmediato. Una complicación intraoperatoria mayor consistió en desgarro de la arteria renal durante la colocación de un clip, por lo cual hubo necesidad de conversión a cirugía abierta. En un paciente con un tumor de $4,5 \mathrm{~cm}$ del polo superior del riñón izquierdo, se presentó lesión del bazo que requirió esplenectomía. Las complicaciones postoperatorias fueron un hematoma de pared, un caso de íleo prolongado y un caso de insuficiencia renal aguda. En estos tres casos se realizó manejo médico y la evolución fue satisfactoria. En los 5 pacientes el tamaño del tumor renal fue menor de $7 \mathrm{~cm}$ de diámetro mayor.

\section{Nefrectomía parcial: cirugía conservadora}

Se operaron 63 pacientes bajo el concepto de cirugía preservadora de nefronas: a 52 se les practicó resección en cuña y a 11 nefrectomía 
parcial, todos por vía transperitoneal. En 18 casos la técnica utilizada fue laparoscópica pura y en 45 el procedimiento se hizo con asistencia manual. Se realizó control de pedículo renal en 24 pacientes. En estos casos, el control vascular se logró clampeando la arteria y vena renal selectivamente. Se presentaron dos complicaciones intraoperatorias y tres complicaciones del postoperatorio temprano. Dos pacientes presentaron sangrado intraoperatorio no controlable que obligó a realizar nefrectomía radical laparoscópica en el mismo acto quirúrgico. Hubo un caso de fístula urinaria en el postoperatorio temprano de un paciente de 56 años, monorreno, que llevó a la formación de un urinoma; esto se resolvió con la colocación de un catéter ureteral de auto-retención en doble $\mathrm{J}$ y punción percutánea de la colección. Un paciente de 34 años de edad, sin factores de comorbilidad, presentó embolismo pulmonar que requirió terapia anticoagulante. En el posquirúrgico tardío este paciente presentó hemoperitoneo y requirió nefrectomía de urgencia. Finalmente, un paciente tuvo un hematoma retroperitoneal sobreinfectado el cual evolucionó satisfactoriamente con drenaje percutáneo y terapia antibiótica.

Todos los especímenes quirúrgicos fueron enviados a estudio histopatológico por congelación, encontrando en dos casos, márgenes quirúrgicos tumorales. El manejo en estos dos pacientes fue la re-resección de los márgenes en el mismo acto quirúrgico. De esta manera, no se tiene ningún caso de márgenes quirúrgicos positivos en el estudio histopatológico definitivo.

Igual que en los pacientes llevados a nefrectomía radical, la extracción de la pieza quirúrgica se hace completa, sin morcelar, en una bolsa de polietileno, lo cual se traduce en el hecho que no se tenga ningún caso de siembras tumorales en las incisiones de extracción de la pieza.

\section{Nefroureterectomía radical}

Dieciséis pacientes con diagnóstico de carcinoma de células transicionales del tracto urinario superior fueron sometidos a nefroureterectomía radical. En todos los casos, el abordaje fue transperitoneal. En este grupo en particular, no hubo complicaciones quirúrgicas.

\section{Nefrectomía simple}

De los 147 pacientes llevados a nefrectomía por patología benigna en 129 se hizo abordaje transperitoneal y en 18 el acceso fue retroperitoneal (lumboscópico). En total hubo 7 complicaciones derivadas de la cirugía: un caso de sangrado a partir del pedículo renal que requirió conversión a cirugía abierta; tres pacientes presentaron sangrado postoperatorio por lo cual tuvieron que ser reoperados, dos de ellos mediante laparotomía y uno con técnica laparoscópica; se presentaron 3 casos de hematoma retroperitoneal: dos pacientes se manejaron médicamente y uno requirió punción percutánea. En todos los casos la evolución fue satisfactoria. En 5 de estos 7 pacientes que presentaron complicaciones, la indicación de cirugía estuvo asociada a un proceso inflamatorio, y hubo un caso asociado a cirugía abdominal previa: pieloplastia laparoscópica.

\section{Heminefrectomía y Nefroureterectomía simple}

Se realizaron 8 heminefrectomías, todas con técnica laparoscópica pura por vía transperitoneal, indicadas por ectopia ureteral y ureterocele con hidronefrosis secundaria. En 7 de los 8 pacientes el sistema afectado fue el superior $y$ hubo un caso indicado por reflujo al sistema inferior. No hubo complicaciones quirúrgicas ni en el postoperatorio inmediato, destacando una adecuada función del parénquima renal no removido. Tampoco hubo complicaciones en los pacientes llevados a nefroureterectomía por patología benigna.

Nefrectomía de donante vivo para trasplante

Se realizaron 23 nefrectomías de donante vivo, todas realizadas por vía transperitoneal con asistencia manual. Se presentó una complicación en este grupo, fue el deslizamiento de un clip de titanio de la arteria renal, lo cual se manejó exitosamente a través del mismo acceso laparoscópico.

La tasa de complicaciones fue del 5,64\% en 319 nefrectomías laparoscópicas consecutivas. No hubo casos de mortalidad en la serie. En la Tabla 1 se describe la distribución de complicaciones de acuerdo al procedimiento realizado y la Tabla 2 muestra la frecuencia de cada una de las complicaciones. 
Tabla 1

Distribución de pacientes llevados a nefrectomía laparoscópica de acuerdo al procedimiento quirúrgico y complicaciones

\begin{tabular}{lccc}
\hline \multicolumn{1}{c}{ Procedimiento } & Número & Complicaciones \\
Nombre & 124 & 7 & Porcentaje \\
\hline Nefrectomía Simple & 70 & 5 & 6,64 \\
Nefrectomía Radical & 63 & 6 & 7,14 \\
Nefrectomía Parcial & 23 & 1 & 7,93 \\
Nefrectomía de Donante & 16 & 0 & 4,34 \\
Nefroureterectomía Radical & 15 & 0 & 0 \\
Nefroureterectomía Simple & 8 & 0 & 0 \\
Heminefrectomía & 319 & 18 & 0 \\
Total & & & 5,64 \\
\hline
\end{tabular}

En nuestra serie, no hubo diferencia estadísticamente significativa en la tasa de complicaciones por sexo, edad o vía de abordaje. El realizar una técnica laparoscópica pura o con asistencia manual, no representó cambio en la tasa de complicaciones. Respecto a las complicaciones relacionadas con el acceso laparoscópico, se presentó un único caso, el cual fue un hematoma de la pared abdominal por lesión vascular durante la colocación de un trocar. Solo hubo un paciente con una complicación derivada del neumoperitoneo: un caso ya descrito de trombo embolismo pulmonar durante una nefrectomía parcial.

Tabla 2

Frecuencia de las complicaciones quirúrgicas en pacientes llevados a nefrectomía laparoscópica

\begin{tabular}{lcc}
\hline Complicaciones & Número & Porcentaje \\
\hline Sangrado & 8 & 2,50 \\
Hematoma Retroperitoneal & 4 & 1,25 \\
Hematoma de Pared & 1 & 0.31 \\
Lesión Esplénica & 1 & 0,31 \\
Trombo Embolismo Pulmonar & 1 & 0,31 \\
Fístula Urinaria & 1 & 0,31 \\
Îleo Prolongado & 1 & 0,31 \\
Insuficiencia Renal Aguda & 1 & 0,31 \\
Total & 18 & 5,64 \\
\hline
\end{tabular}

\section{DISCUSIÓN}

De las intervenciones urológicas realizadas con técnica laparoscópica, la nefrectomía es una de las más comúnmente practicadas $^{5}$. Existen pocas complicaciones específicas de la laparoscopia y el riesgo de que éstas ocurran, es muy bajo: se estima que en sólo 0,3 a 3 de cada 1.000 intervenciones se presenta una complicación grave, como lesión vascular o intestinal, derivada de la punción inicial, la insuflación o la colocación del primer tro$\operatorname{car}^{5,6}$. Otras complicaciones como lesiones vasculares o intestinales, eventos cardiovasculares, pulmonares, embólicos y alérgicos, que ocurren en el acto quirúrgico y período postoperatorio, también se presentan en cirugía abierta, en una proporción similar a la técnica laparoscópica ${ }^{5,7}$.

La mayor parte de las complicaciones en cirugía laparoscópica ocurren durante las primeras intervenciones de la curva de entrenamiento de un cirujano; en la medida que se obtiene experiencia, el porcentaje de estos eventos disminuye notoriamente $^{4,5,8-11}$ (Tabla 3). En la Figura 3 se muestra el número anual de nefrectomías y complicaciones en nuestra serie. Hubo una tendencia al aumento en el número de procedimientos laparoscópicos, mientras que la tasa de complicaciones se mantuvo en igual proporción. La tasa de complicaciones no disminuyó en el tiempo, debido a que se realizaron cada vez más cirugías de mayor complejidad (Fig. 4). La nefrectomía parcial por tumor, considerada en la literatura como

Tabla 3

Complicaciones de la nefrectomía laparoscópica en diferentes series

\begin{tabular}{lcc}
\hline $\begin{array}{l}\text { Autor } \\
\text { (Referencia) }\end{array}$ & $\begin{array}{c}\text { \% Complicaciones en } \\
\text { 100 primeros } \\
\text { casos }\end{array}$ & $\begin{array}{c}\text { \% Complicaciones } \\
\text { después de 100 } \\
\text { casos }\end{array}$ \\
\hline Vallancien (5) & 13,3 & 3,6 \\
Soulie (8) & 9 & 4 \\
Simon (9) & 8,3 & 2,5 \\
\hline
\end{tabular}




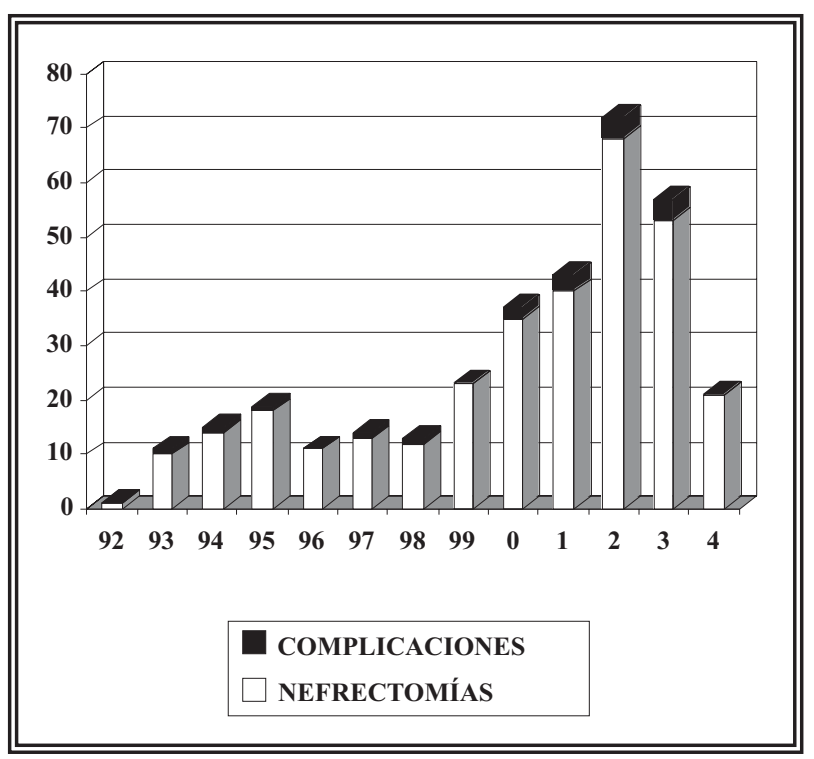

FIGURA 3. Distribución anual de pacientes llevados a nefrectomía laparoscópica de acuerdo a complicaciones.

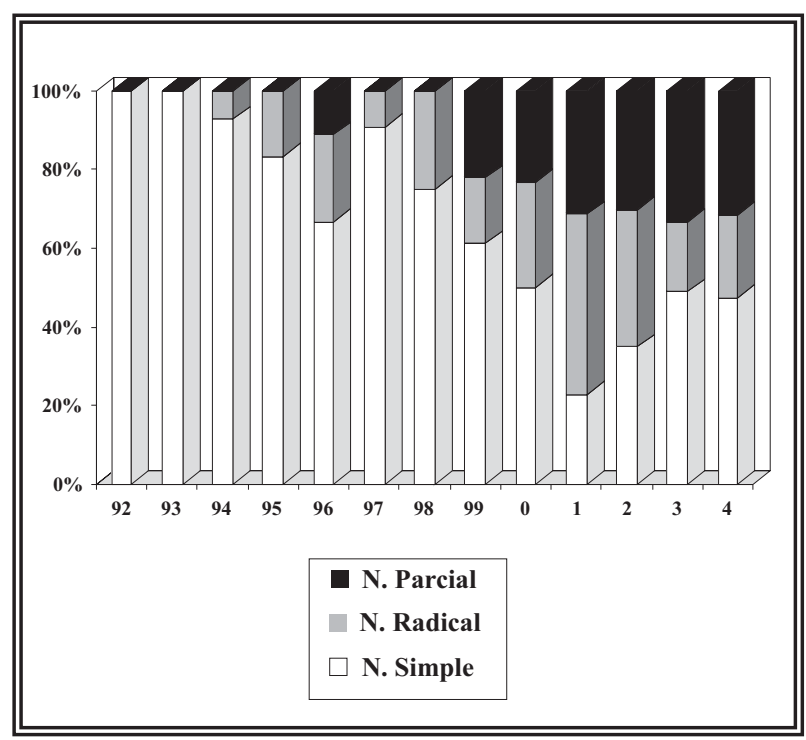

FIGURA 4. Proporción anual de pacientes llevados a nefrectomia simple, nefrectomía radical y nefrectomia parcial por laparoscopia.

procedimiento de alta complejidad, tuvo una baja tasa de complicaciones $(7,9 \%)$, lo cual se explica porque se empezó a realizar cuando ya se contaba con la experiencia adquirida en nefrectomía simple y radical.

A diferencia de lo informado en otras series ${ }^{5,9-10}$, en la nuestra no hubo casos de complicaciones derivadas de la posición del paciente en la mesa de operaciones. Vallancien et al. $^{5}$ mencionan varios casos de paresia cubital transitoria y dolor escapular, los cuales se resolvieron en forma espontánea. Ninguno de nuestros pacientes ha manifestado la presencia de estos sintomas.

Existe controversia en la literatura en cuanto a la vía de abordaje para nefrectomía laparoscópica: transperitoneal o lumboscópica, con respecto al riesgo de lesión vascular o de órganos adyacentes y la posibilidad de íleo postoperatorio. En nuestra serie, la mayoría de nefrectomías se realizaron por vía anterior transperitoneal $(94,3 \%)$ encontrando tan sólo un caso de íleo postoperatorio. A pesar del número reducido de procedimientos realizados por vía lumboscópica $(5,7 \%)$, no se encontró diferencia estadísticamente significativa en las complicaciones entre las dos vías de abordaje.

En nuestra experiencia, la tasa de complicaciones fue del $5,64 \%$. Consideramos que es un resultado óptimo, más cuando se compara con otros grupos con experiencia en cirugia laparoscópica (Tabla 4) . La edad promedio de nuestros pacientes fue de 45,1 años, sin embargo, la edad no fue un factor de riesgo para complicaciones en esta serie: no hubo casos de eventos cardiovasculares o tromboembólicos asociados a la edad. El único caso de trombo embolismo pulmonar se presentó en un hombre de 34 años, sin factores de comorbilidad.

El sangrado fue la complicación más frecuente: 8 casos de sangrado quirúrgico o postoperatorio y 4 casos de hematoma retroperitoneal. En dos pacientes llevados a nefrectomía parcial, hubo sangrado intraoperatorio de difícil control. En estos casos el manejo de la complicación se hizo por vía laparoscópica: nefrectomía radical en el mismo acto quirúrgico. Tres pacientes fueron reintervenidos por sangrado en el postoperatorio inmediato: dos de ellos mediante laparotomía, y el último paciente que se reintervino por sangrado, se hizo con técnica laparoscópica, con resultado satisfactorio.

\section{CONCLUSIONES}

Nuestra serie de nefrectomía laparoscópica muestra una baja tasa de complicaciones, acorde con los diferentes reportes de la literatura. Consideramos que en manos experimentadas, la laparoscopia es una técnica segura en pacientes que requieren cirugía renal ablativa. 
Tabla 3

Series de nefrectomía laparoscópica según autor.

\begin{tabular}{lcccc}
\hline Autor & No Pacientes & \% Complicación & \% Conversión & \% Mortalidad \\
\hline Gill, 1995 & 185 & 16 & 5 & 0 \\
Soulie, 2001 & 20 & 5 & 5 & 0 \\
Vallancien, 2002 & 162 & 12 & 7 & 0 \\
Siqueira, 2002 & 213 & 7,5 & 6,1 & 0,5 \\
Simon, 2004 & 285 & 5,6 & 4 & 0 \\
Serie actual, 2005 & 319 & 5,6 & 1,3 & 0 \\
\hline
\end{tabular}

La tasa de complicaciones no se modificó por la edad, la vía de abordaje: transperitoneal o lumboscópico, ni por la técnica utilizada: laparoscópica pura o con asistencia manual. La experiencia acumulada del grupo permitió realizar cirugías cada vez más complejas sin aumentar la tasa de complicaciones, llegando incluso a realizar el manejo de algunas de estas complicaciones, también mediante técnica laparoscópica.

\section{REFERENCIAS}

1. Clayman R, Kavoussi LR, Soper NJ, Dierks SM, Meretyk S, Darcy MD, et al. Laparoscopic nephrectomy: initial case report. J Urol. 1991;146(2):278-281.

2. Castillo O, Van Cauwelaert R, Wohler C. Nefrectomía Laparoscópica: experiencia en 20 pacientes. Rev Chil Cir. (Chile). 1995; 47:235-239.

3. Castillo O, Portalier P, Diaz M, et al. Nefrectomía laparoscópica en niños. Rev Chil Pediatr. (Chile). 2002;73:495-499.

4. Gill IS. Laparoscopic radical nephrectomy for cancer. Urol Clin North Am. 2000;27(4):707-719.

5. Vallancien G, Cathelineau X, Baumert H, Doublet JD, Guillonneau B. Complications of transperitoneal laparoscopic surgery in urology: review of 1311 procedures at a single center. J Urol. 2002; 168(1):23-26.
6. Witz M, Lehmann J. Major vascular injury during laparoscopy. Br J Surg. 1997;84(6):800-804.

7. Dunn MD, Portis AJ, Shalhav AL, Elbahnasy AM, Heidorn C, McDougall EM, et al. Laparoscopic versus open radical nephrectomy: a 9 year experience. J Urol. 2000;164(4):1153-1159.

8. Soulie M, Seguin P, Richeux L, Mouly P, Vazzoler N, Pontonnier F, et al. Urological complications of laparoscopic surgery: experience with 350 procedures at a single center. J Urol. 2001;165(6 Pt 1):1960-1963.

9. Simon SD, Castle EP, Ferrigni RG, Lamm DL, Swanson SK, Novicki DE, et al. Complications of laparoscopic nephrectomy: The Mayo Clinic experience. J Urol. 2004;171(4):1447-1450.

10. Siqueira TM Jr, Kuo RL, Gardner TA, Paterson RF, Stevens LH, Lingeman JE, et al. Major complications in 213 laparoscopic nephrectomy cases: The Indianapolis experience. J Urol. 2002;168(4 Pt 1):1361-1365.

11. Gill IS, Kavoussi LR, Clayman RV, Ehrlich R, Evans R, Fuchs G, et al. Complications of laparoscopic nephrectomy in 185 patients: a multi-institutional review. J Urol. 1995; 154(2 Pt 1):479-483.

Dr. O. Castillo Cádiz

E-mail: octaviocastillo@vtr.net

(Trabajo recibido el 8 de septiembre 2005) 